\title{
Fukushima: lecciones japonesas para la Salud Pública colombiana.
}

\author{
Omar Segura, MD-PhD, FETP ${ }^{1,2}$ osegura@smc-as.com \\ 1. Doctor en Salud Pública, Universidad Nacional de Colombia \\ 2. Asesor Científico, SMC-AS Unidad de Investigaciones, Bogotá - Colombia
}

Fecha de recepción: 12/12/2018

Fecha de corrección 02/02/2019

Fecha de aprobación 20/04/2019

Fecha de publicación 30/06/2019

\begin{abstract}
Resumen
Introducción: Durante el 21o. Congreso Mundial de Epidemiología, organizado por la Asociación Internacional de Epidemiología (IEA) en Saitama-Japón en agosto de 2017, se aceptó invitación de los organizadores para visitar la Planta Nuclear \# 1 de Fukushima. Objetivo: Explorar la situación operacional y de salud pública en el área de la prefectura de Fukushima y su planta nuclear\#1, sitio afectado por el terremoto y tsunami de 2011. Métodos: Salida de inspección de campo (Inspection Tour) y encuentro con representantes de TEPCO (Tokyo Energy Power Company) en área de control; explicación didáctica de antecedentes y situación actual zonal, e instrucciones (briefing) para acceso. Visita con explicación de zonas de atención de salud y trabajos realizados. Recorrido en autobús especialmente adaptado al perímetro de cada reactor, portando dosímetro individual. Reunión final para resolución de dudas e inquietudes y lecciones del caso.
\end{abstract}

Resultados: Visita y reuniones ejecutadas; exposición personal $=$ $0,01 \mathrm{mSv} / \mathrm{h}$. Radiación por zonas de reactor: central $=230 \mathrm{mSv} / \mathrm{h}$; paredes $=40 \mathrm{mSv} / \mathrm{h}$; planta $=0,26 \mathrm{mSv} / \mathrm{h}$, periferia $=0,26 \mathrm{mSv} / \mathrm{h}$. Zona de exclusión $=20 \mathrm{~km}$. Políticas de salubridad basadas en limpieza y remoción de fuentes de contaminación; aislamiento de aguas y bombeo de fuentes subterráneas; prevención y contención de escapes.

Conclusión: La prevención lo es todo en asuntos industriales, sean nucleares o no; la colaboración interpersonal e interagencias es esencial en el manejo a muy largo plazo de desastres similares; debe evitarse incorporar técnicas o tecnologías industriales desconocidas o foráneas sin antes valorar su costo, alcance y posibles riesgos de salud individual, pública o medio-ambiental.

Palabras clave: Salud Pública, Epidemiología de desastres, Liberación de Radiactividad Peligrosa, Protección Ambiental, Vigilancia Sanitaria Ambiental.

Copyright (C) Facultad de Ciencias de la Salud de la Universidad Tecnológica de Pereira. 1995-2018. Todos los derechos reservados.
Fukushima: japanese lessons to colombian Public Health.

\begin{abstract}
Introduction. During the 21st. International Epidemiology Association (IEA) World Congress of Epidemiology at Saitama (Japan), celebrated in August 2017, an invitation from its organizers to visit Fukushima Nuclear Plant \# 1 was accepted. Objective: To explore operational and public health situation at the area of the Fukushima Prefecture and its nuclear plant \#1, place affected by the 2011 earthquake and tsunami.

Methods. Inspection tour and appointment with representatives of TEPCO (Tokyo Energy Power Company) at the control area; explanation on background and actual situation, and briefing before access. Guided visit to areas dedicated to healthcare and workers' attention, and work done. Tour by specially adapted autobus to the perimeter of each reactor, using individual dosimeter. Final meeting to solve questions and lessons of this case.
\end{abstract}

Results. Visit and meetings were executed; individual exposure $=0,01 \mathrm{mSv}$. Radioactivity by reactor zones: central (inside) $=230$ $\mathrm{mSv} / \mathrm{h}$; walls $=40 \mathrm{mSv} / \mathrm{h}$; plant $=0,26 \mathrm{mSv} / \mathrm{h}$, perimeter $=0,26 \mathrm{mSv} / \mathrm{h}$. Exclusion zone $=20 \mathrm{~km}$. Public Health policies in place based upon cleansing and removal of sources of contamination; water isolation and subterranean sources pumping; prevention and containment of leaks.

Conclusion: About industrial issues, prevention is everything, whether they be nuclear or not; interpersonal / interagencies' cooperation is paramount when dealing with similar disasters at a very long term; unknown or poorly understood industrial techniques or technologies should be avoided before a proper evaluation of their risk/benefit balance, scope and possible health risks to subjects, communities or environment.

Keywords: Public Health, Disaster Epidemiology, Radioactive Hazard Release, Radioactivity Release, Environmental Protection, Environmental Health Surveillance.

Copyright (C) Facultad de Ciencias de la Salud de la Universidad Tecnológica de Pereira. 1995-2018. Todos los derechos reservados.

\section{Introducción}

El accidente de la planta nuclear \# 1 de Fukushima, ocurrido en marzo 11 de 2011, fue consecuencia directa de un terremoto de magnitud 9.0 y subsecuente tsunami, que afectó a todo el Japón y en particular a su zona noreste, la región de Tōhoku. Una cascada de fallas de equipo en varias unidades de dicha planta llevó a la liberación de material radiactivo en la atmósfera y en el mar; los niveles de radiactividad, inicialmente de $12,000 \mathrm{mSv} / \mathrm{h}$ a expensas de la precipitación de isótopos como ${ }^{131} \mathrm{I}(160 \mathrm{PBq})$ y ${ }^{137} \mathrm{Cs}(36.7 \mathrm{PBq})$ disminuyeron en mayo de 2011 a $350 \mathrm{mSv} / \mathrm{h}$. 
El gobierno japonés, con base en estos datos, implementó medidas de emergencia para proteger la población de la zona, tales como evacuación con perímetro de exclusión de $20 \mathrm{~km}$, prohibición de venta y consumo de comida contaminada, distribución de tabletas de yodo e inicio de las operaciones de descontaminación y cese de operaciones de la planta $(1,2)$. Estudios posteriores de la zona han encontrado aberraciones genéticas, en biológicas y morfológicas en especímenes vegetales y animales en los bosques circundantes (3).

La Asociación Internacional de Epidemiología (IEA) organizó su 21․ Congreso Mundial para realizarse en la ciudad de Saitama (Japón) en agosto de 2017. Uno de los temas centrales en el evento fueron los efectos ya tardíos y a muy largo plazo de la detonación de dos bombas atómicas en Hiroshima y Nagasaki en 1945, y también aquéllos a corto plazo como consecuencia de la catástrofe. Participando como expositor de dicho evento, el suscrito recibió una invitación de los organizadores a todos los de profesionales y científicos asistentes al congreso para realizar una visita de inspección a la Planta Nuclear \# 1 de Fukushima.

Los motivos para aceptar dicha invitación fueron de índole personal y profesional, dados la propia curiosidad y el deseo de conocer de primera mano y por los propios afectados qué fue lo que pasó, cómo lo han manejado y qué esperan hacia el futuro; y que el artículo 81 de la Constitución Nacional colombiana de 1991 prohíbe fabricar, importar, poseer y usar armas nucleares, así como introducir al territorio nacional residuos nucleares, lo que en últimas equivale a una prohibición para contar con plantas nucleares en suelo colombiano. Por tanto, el objetivo fue explorar la situación operacional y de salud pública en el área de la prefectura de Fukushima y su planta nuclear \#1.

\section{Métodos}

Este constituye un reporte de visita de campo de un día de duración (2018-08-23), que comenzó a las 06:00 con la reunión inicial del grupo de visitantes, salida de inspección (Inspection Tour) y desplazamiento por tierra desde la ciudad de Saitama hasta el área de control situada a 20 $\mathrm{km}$ de la planta (enerugi kan) para el encuentro a las 10:00 con representantes de TEPCO (Tokyo Energy Power Company). Dichos funcionarios ofrecieron a los participantes una explicación didáctica (briefing) de los antecedentes y la situación actual en la planta, e instrucciones para realizar debidamente el acceso. Desde el área de control, en un autobús especialmente acondicionado, se ejecutó la visita grupal a la sede de la planta propiamente dicha, entre 11:00 y 13:00. Tanto los funcionarios de TEPCO como los visitantes pasaron los controles de seguridad, para proseguir a un área dedicada a atención inicial de emergencias de salud y radiológicas; allí se recibió una explicación sobre su funcionamiento y los trabajos realizados. Después de esto, se ejecutó un recorrido en autobús especialmente adaptado diferente al de ingreso y portando un dosímetro individual al perímetro de cada reactor y un chaleco para sujetarlo. Tras este recorrido, de una hora de duración, todo el grupo dejó las instalaciones de la planta y volvió al área de control, donde los funcionarios de TEPCO ofrecieron una reunión final a las 13:00 para atender y resolver dudas e inquietudes de los visitantes respecto a las medidas tomadas, los planes futuros de desactivación de planta y las lecciones del caso. El retorno a Saitama ocurrió sobre las 18:30.

\section{Resultados}

Figura 1 Ubicación de planta nuclear

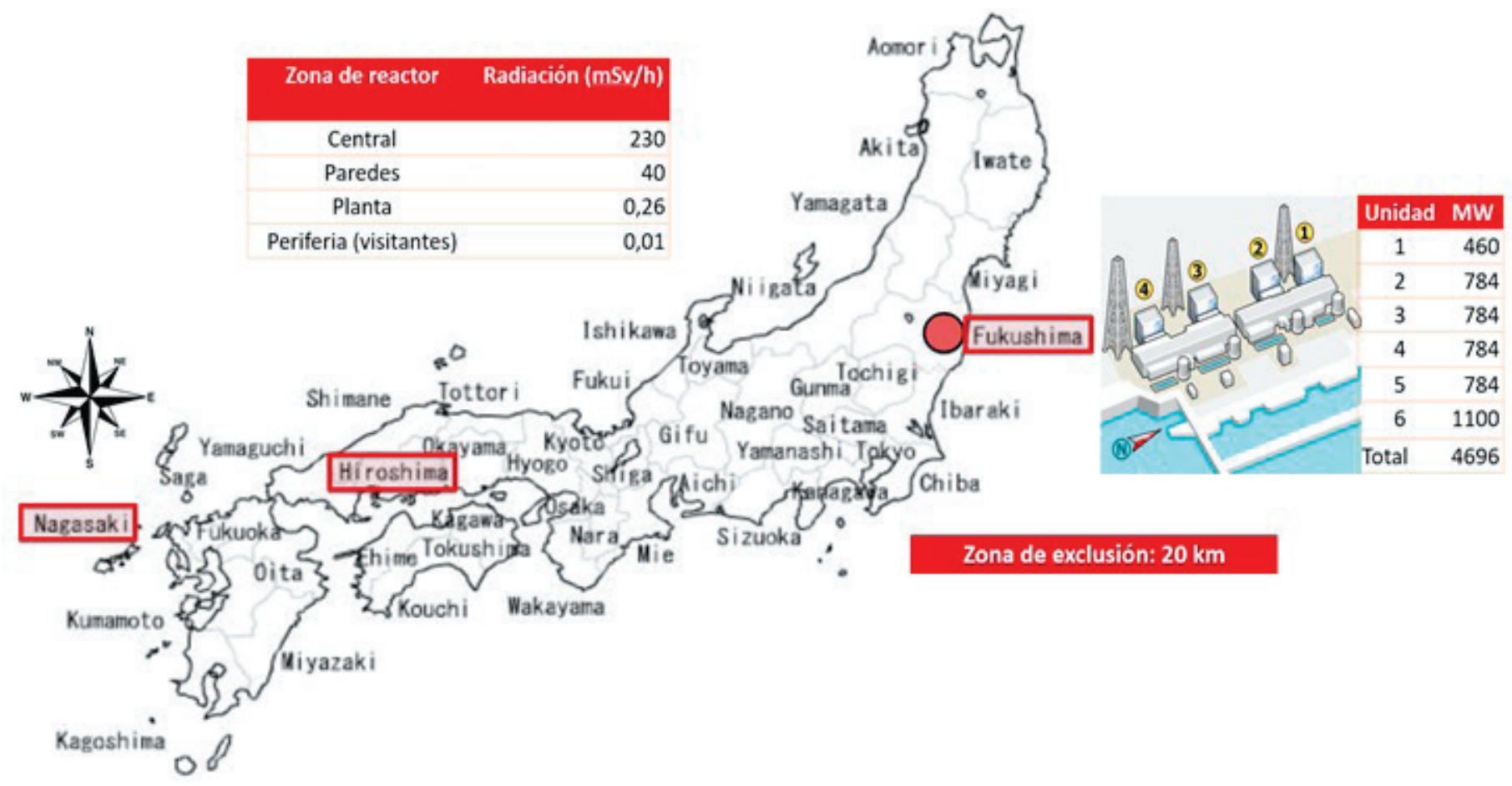

Bajo coordinación general del secretario del congreso, dr. Toshiyuki Ojima, se ejecutó una salida de inspección de campo (Inspection Tour) 
a la planta el día 23 de agosto de 2017, en la que participaron 15 personas, locales y extranjeras. Las plantas nucleares de Fukushima \# 1 y \# 2 se encuentran en el municipio de Ōkuma, en la prefectura de Fukushima, a unos $265 \mathrm{~km}$ al noreste de Tokyo. La planta \# 1 tiene un área de $3,5 \mathrm{~km}^{2}$, y cuenta con seis reactores, todos actualmente desactivados (Figura 1).

El encuentro con representantes de TEPCO estaba programado en un área de control a $20 \mathrm{~km}$ de la planta propiamente dicha. Esta área de control se encuentra en el límite de la zona de exclusión delimitada por el gobierno japonés, ésta última dispuesta en su momento para evacuación y contención del desastre. La primera cosa impactante al llegar fue constatar cómo existe una línea imaginaria pero muy palpable de división entre "área segura" y "área insegura", la diagonal entre una iglesia y un exposímetro, cuyas cifras eran: 0,261 $\mathrm{mSv} / \mathrm{h}$. El grupo fue atendido, con la habitual amabilidad japonesa, por funcionarios del área de Relaciones Públicas de TEPCO; uno de ellos, quien fue miembro del equipo de emergencias pero no de los llamados "Cincuenta de Fukushima" (Fukushima 50) atendió al grupo.

Es proverbial la forma como son los salones de clase japoneses: ciertamente con un aire occidental, pero con pisos de vinilo, remate de pared tipo tercio de caña, pintura basada en aceite a las paredes, pero sobre todo limpio, de aspecto nuevo y bien mantenido (así tenga muchos años), y además el respeto al docente: todos de pie antes de comenzar la sesión. Los funcionarios realizaron una explicación didáctica (briefing) de los antecedentes y la situación actual en la planta.

\section{Fig 2. Ubicación de los reactores}

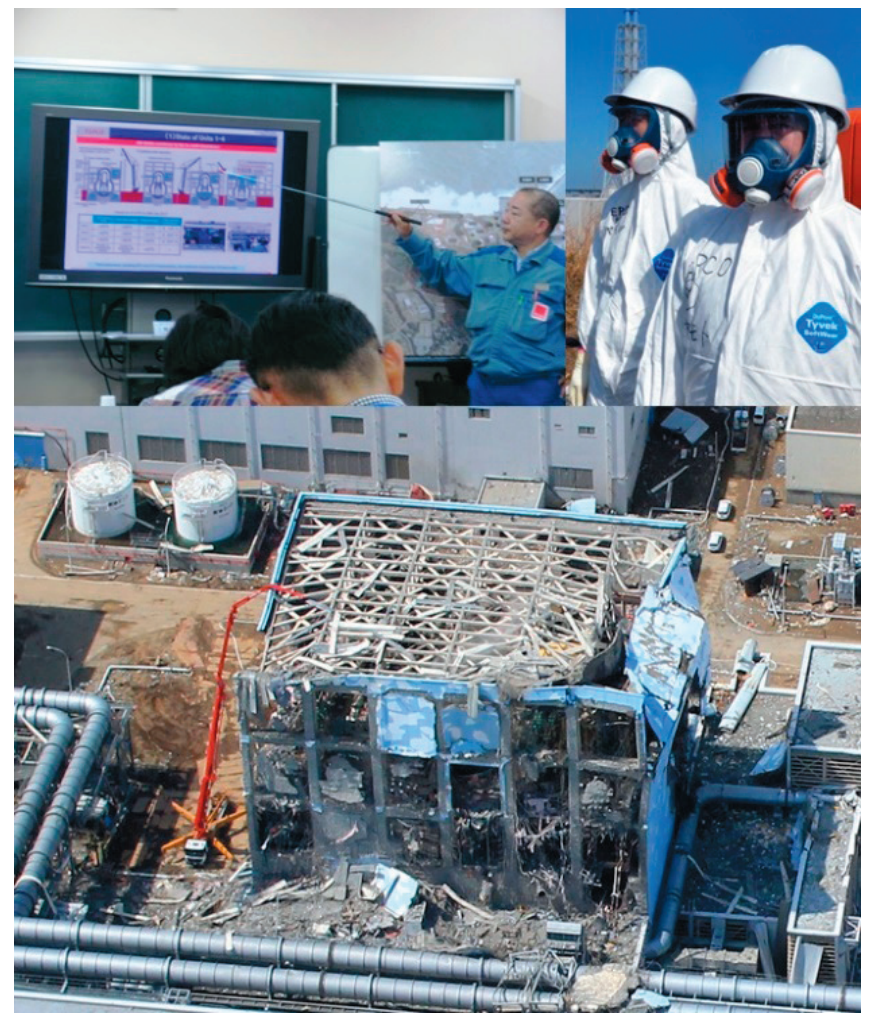

El relacionista de TEPCO (figura 2) nos informó cuál era la situación de los famosos cuatro reactores en la planta Nuclear \# 1 - realmente son seis, divididos en un área de dos y otra de los cuatro famosos, y en la zona son realmente dos plantas, la Daiichi (la \# 1) y la la Daini (la \# 2). Esencialmente, la planta presentó fundición del material nuclear en tres de los cuatro reactores; uno de ellos, el reactor \# 2, estaba apagado el día de la tragedia, y lo que han hecho con él es remover el combustible nuclear. La traductora fue vertiendo en un ágil y excelente inglés la explicación en japonés, que básicamente se centró en las medidas para reciclar el agua, manejar y disponer de los desechos contaminados. Mientras éramos informados, otro grupo de TEPCO verificó nuestros pasaportes o tarjetas de identificación locales. Sin gritos ni amenazas, sin armas ni despliegues de fuerza, todo con absoluto respeto y precisión casi militar.

Evitando referirse directamente al terremoto y tsunami de 2011, y a diversas críticas que TEPCO y el propio gobierno de ese país recibieron su momento, explicaron directamente la estructura de la planta, el poder generador de cada reactor, el manejo que se venía dando con equipo súper-pesado de ingeniería para la remoción de escombros, especialmente del reactor \# 3 , políticas de salud pública y manejo en lo tocante a material radioactivo, desechos y seguridad ocupacional para el personal. Al final de esta charla se recibieron instrucciones para acceso, consistentes esencialmente en dejar atrás y bajo custodia todo material de fotografía, metálico y no esencial, y utilizar la propia ropa si ésta cubría toda superficie corporal salvo la cara; a algunos compañeros de visita que tenían ropa ligera o de verano les fue provista chaqueta o pantalón. Los funcionarios de TEPCO fueron muy claros en que no se permitían grabaciones ni videos ni fotos en el lugar, pero sí podíamos dejar nuestras cosas en el salón, que sería cerrado y asegurado.

Una vez recibida la explicación, el grupo se desplazó en un bus diferente $20 \mathrm{~km}$ al norte, al área del nuevo edificio de TEPCO, ya en las instalaciones de la planta. Formados todos los visitantes, nos fue entregada una tarjeta amarilla con collar negro que, en japonés, explicaba el carácter de visitante externo, y se hizo la entrada por un sistema de seguridad idéntico al usado para acceder al embarque de aviones; esto es, poner todo objeto de metal en bandejas que son escaneadas, y cruzar por un escáner corporal convencional.

Cumplido esto, el grupo pasó a un cuarto donde, a la manera japonesa, todos nos quitamos los zapatos; este salón era la "Sala de Emergencia". En ella, el respectivo jefe de área informó que tenían toda clase de equipo médico (incluso había elementos como "Little Anne", maniquí usado para enseñar y entrenar resucitación cardiopulmonar); explicó que tienen articulación con las autoridades municipales y prefecturales (la prefectura en Japón es como un estado en México o un departamento en Colombia) para trasladar, si fuese necesario en helicóptero, cualquier trabajador o visitante que manifieste síntomas de posible envenenamiento radiactivo. El jefe de área dejó claro que sí habían tenido accidentes ocupacionales, unos 30 , pero relacionados con situaciones de congelación o golpe de calor, según la estación del año; fueron muy enfáticos en responder, al ser interrogados, que no habían tenido accidentes ocupacionales por radiactividad sino más bien por esas situaciones, con cuatro incidentes laborales en 2017, todos relacionados con deshidratación o fatiga física; esta información se confirmó independiente y posteriormente (4).

Las personas que trabajan ya en la zona de los reactores utilizan diversos tipos de protección radiológica, destacando dosímetros, ropa aislante Tyvek ${ }^{\circledR}$ y máscaras de respiración, con explicación de zonas de atención de salud y de trabajos realizados (figura 2).

A continuación, el grupo pasó a otro salón, con sillas organizadas de a cinco en fondo, que contenían los dosímetros. Estos aparatos son muy similares en aspecto a un buscapersonas (beeper) de color rosado, pero el doble o triple de pesados, con una abertura detectora y una pequeña pantalla lectora que da la lectura de radiación en $\mathrm{mSv} / \mathrm{h}$. Según la explicación, $0,01 \mathrm{mS} / \mathrm{h}$ es equivalente, en condiciones de limitada exposición en el tiempo, a unos rayos X de tórax. Por 
todos lados uno veía tablas de conversiones, equivalencias, siempre al estilo japonés con anime. Los visitantes recibieron un chaleco protector para guardar el dosímetro, colgado del collar, y de uno en uno y en fila india subimos al autobús para el recorrido. El verano japonés es caluroso y húmedo; había un calor de $32 \mathrm{C}$ pero muy seco, casi sin viento. Inicialmente creíamos que íbamos a necesitar ropa protectora blindada, o cuando menos un traje Tyvek ${ }^{\circledR}$ con máscara. Fue innecesario: portando el mencionado dosímetro y sin ninguna protección adicional a la mencionada se realizó el recorrido por cada zona perimetral de los reactores. Este recorrido se realizó con los funcionarios de TEPCO acompañados de una traductora, y duró una hora.

Nada puede igualar esa sensación de silencio sobrecogedor, de ausencia total de sonidos, de destrucción en reactores encajonados en cubos de unos 100 metros de arista; las vías de acceso están ubicadas a 35, 15 y 10 metros sobre el nivel del mar, se ven las marcas del tsunami tanto en la pintura como en la compresión y atrición de varios tanques de almacenamiento, cada uno para 1.000 toneladas, aplastados por su base como si fuese la mano de un gigante de agua triturando una lata de cerveza. Se apreciaron los destrozos en el área del reactor \#2 (figura 2); el nivel de radiación alcanza ahí dentro los $230 \mathrm{mSv} / \mathrm{h}$, mientras que en el tejado y muros llega a los $40 \mathrm{mSv} / \mathrm{h}$, pero en las vías que el bus recorría, con sus sillas forradas en plástico, es de $0,06 \mathrm{mSv} / \mathrm{h}$. La exposición personal fue de $0,01 \mathrm{mSv} / \mathrm{h}$.

Quepa decir que la radiación, igual como pasa en Chernobyl o en Three Mile Island, se reparte de forma desigual; no son círculos concéntricos perfectos como los usados para explicar a la gente los efectos de un arma nuclear. Los ingenieros de TEPCO, Hitachi y Toshiba aprovecharon las redes de tubería que servían para irrigar la planta, y construyeron nuevas redes, para tratar de congelar el suelo a $-30{ }^{\circ} \mathrm{C}$, para así amortiguar el calor que posee el material fundido adentro, para limitar el daño al subsuelo y para facilitar las operaciones de limpieza y desmantelamiento, pues TEPCO debe cerrar la planta por una decisión política desde Tokyo, y limitar el alcance de los daños, tarea que le puede tomar, como a sus colegas rusos, entre 30 y 50 años.

Mientras que los funcionarios de TEPCO nos acompañaron en el bus y nos traducían, las explicaciones fueron ágiles y comprensibles; el retorno, como la posterior salida, fue en absoluto silencio.

La salida del área fue igual que la entrada, por autobús. Al bajar, entramos a un recinto que albergaba una suerte de máquinas detectoras de radiación a la manera de escáneres corporales de espacio estrecho, cerrados, activados con la presión de las manos para abrir la puerta del otro lado si el escaneo iba bien. Aunque en el horario y plan de actividades esperadas estaban contemplados exámenes físicos y de screening, no ocurrió nada de eso, sino que el grupo de visitantes salió y pasó a un área contigua donde se devolvieron los carnets, y salió en el autobús.

Finalizado el recorrido, el grupo regresó al área de control, para resolver dudas e inquietudes respecto a las medidas tomadas, planes futuros de desactivación de planta y lecciones que dejó el caso al pueblo, empresarios y gobierno nipones. Tanto en el briefing precedente como en este momento, fueron permitidas fotografías esquemáticas de la planta y grabaciones de audio/video a los funcionarios.

Las preguntas fueron tan numerosas como extensas fueron las explicaciones de los relacionistas de TEPCO: por ejemplo, ante la pregunta del suscrito sobre cuál era la lección de países con energía nuclear como Japón para pueblos como el colombiano que no la usa y la tiene prohibida, contestaron que en efecto habían hecho todos los cálculos matemáticos y de ingeniería y llegaron a establecer o a asumir el diseño bajo un terremoto/tsunami 8,0 y una ola de $10 \mathrm{~m}$ de alto; jamás llegaron a imaginar que enfrentarían un terremoto de un orden de magnitud mayor (la escala de terremotos es logarítmica) y un tsunami con un frente de ola de 15 metros de alto; finalmente, la conclusión fue: "la prevención lo es todo en este negocio". Hubo varias preguntas técnicas, referentes al retiro del material fundido del reactor, a las proporciones de elementos radiactivos, o al manejo del agua contaminada con tritio (la llamada agua superpesada); fue llamativo sentir en todo momento y en sus respuestas el espíritu de trabajador duro y persistente nipón, simbolizado en la expresión Ganbatte kudasai! (traducible en nuestro entorno como ¡Insistir, Resistir y Persistir!), pues desconocen muchas cosas, los reactores les siguen "jugando sucio", pero persisten buscando soluciones a problemas nuevos que les ha suscitado este desastre único en el mundo entero.

Saliendo de la zona a las 14:00, el retorno fue más distendido, más amable, pero guardando la apropiada distancia explícita en el trato amable y educado de los nipones, quienes -al menos en este grupoentienden el inglés pero prefieren hablar en japonés y contar con traductora en visitas o salidas de este talante.

\section{Discusión}

Se destaca, en primer lugar, no sólo de esta visita de inspección sino de todas las circunstancias de viaje del suscrito, la organización, la persistencia y la disciplina que el pueblo japonés le pone a todos sus empeños, empresas y cometidos. Son absolutamente puntuales, buscan ser absolutamente claros en instrucciones y en procederes, y sobre todo absolutamente honestos en lo que quieren y pueden ofrecer. Por supuesto que tienen su naturaleza humana, no son las máquinas o robots que ellos diseñan y construyen, y son amables con el visitante extranjero tanto como son realmente ásperos con el que quiere quedarse, pero sí tienen estos valores incorporados en su cultura y proceder habituales. Para dar una idea a los lectores, no se halló un solo hueco o defecto de la autopista o sus largos túneles, a veces de 2 a $4 \mathrm{~km}$, durante todo el trayecto, y ni siquiera hubo material de construcción o escombros caídos en las zonas de recorrido perimetrales a los reactores. Todo en un marco de absoluta limpieza y organización que recuerda mucho la aplicación de la estrategia $5 \mathrm{~S}$, de origen nipón, formalizada por Osada en 1980 y que es conocida en Colombia: Seiri (clasificar), Seiton (ubicar), Seiso (barrer), Seiketsu (estandarizar), Shitsuke (sostener) (5).

En segundo lugar, fue patente el temor de los asistentes al evento respecto de la exposición inmediata y de las consecuencias a mediano y largo plazo por participar en una visita de inspección de este tipo; al respecto, Scholl y cols. (6) ya señalaban en 2013 que los seres humanos estamos expuestos durante nuestra vida a fuentes inescapable de radiación ionizante, tanto de origen terrestre como espacial, en la forma de los elementos pesados que hacen parte de la corteza terrestre o de los rayos cósmicos, lo que traduce en una exposición anual promedio de $2.4 \mathrm{mSv} / \mathrm{año}($ rango $=1-100) \mathrm{y}$ un límite para daño tisular de aproximadamente $100 \mathrm{mSv}$. Como comparación, la dosis de radiación ionizante por unos rayos $\mathrm{X}$ de tórax es de $0,01 \mathrm{mSv} / \mathrm{h}$, y por un vuelo desde Bogotá hasta Tokio de entre 0,1 y $0,2 \mathrm{mSv}$.

Hasegawa y cols (7) y Ohtsuru y cols. (8) y señalan que todos los desastres nucleares, incluyendo los ataques a Hiroshima y Nagasaki en 1945 y los accidentes más graves en plantas nucleares -Kysthym, URSS (1957); Windscale Piles, GB (1957); Three Mile Island, EUA (1979); Chernobyl, URSS (1986) y Fukushima, Japón (2011)- tienen 
similitudes en términos de los efectos sobre la salud pública, sean éstos o no a sociables a la exposición a radiación. Aún cuando son raros (ipor fortuna!), su número, sus circunstancias y la política nacional que sea dominante (típicamente, una de "seguridad nacional") limita procesos rigurosos de investigación más allá del manejo inmediato de la emergencia o catástrofe, trazado de intervenciones y aplicación de estrategias, identificar lecciones a aprender y desarrollar planes que permitan proteger al público, minimizar los efectos negativos y proteger a los trabajadores de la radiación. En todos estos casos, mucho del esfuerzo inicial ha tenido que ver con el cuidado inmediato de daños físicos y de salud mental relacionados con una exposición a radiación y, en mayor medida incluso, con las consecuencias de una evacuación y desplazamiento de larga duración. Queda, sin embargo, el asunto de la comunicación confiable de los riesgos individuales de una catástrofe de este tipo, y el manejo de los efectos sociales y psicológicos, para la cual se precisa entrenamiento para afrontar y responder a este tipo de emergencias que incluye intervenciones basadas en evidencia, investigaciones de campo y apoyo a la toma de decisiones de riesgo/beneficio, y un proceso abierto y conjunto de aprendizaje para la mitigación de futuros desastres.

En suma, ¿Cuáles son las lecciones de un país nuclear (Japón) para un país no-nuclear (Colombia)? Las lecciones de los funcionarios de TEPCO para todos los asistentes fueron las siguientes:

"La prevención lo es todo al diseñar, construir o gestionar cualquier planta o entorno industrial, sea ésta nuclear o no", lo que traduce en contar con valoraciones de riesgo y de imprevistos frente a escenarios de desastre o catástrofe incluso implausibles, construir capacidad de protección medio-ambiental y de comunicación de riesgo $(2,9)$. Esto fue confirmado por uno de los visitantes, el ingeniero David Wroth, de Underwriters Laboratories (UL, www.ul.com) y miembro, en su juventud, del equipo de evaluación técnica y descontaminación de la planta de Three Mile Island.

- "Hay problemas de salud pública más allá de la radiación", que pasan por el acceso a agua, comida, medicamentos e insumos, la comunicación de riesgos individuales, el manejo de pérdidas y duelos, la recuperación de sectores económicos e industriales frente a una población evacuada en desplazamiento de larga duración, entre otros $(8,10)$.

4 "Son vitales la protección individual, comunitaria, institucional y medioambiental.", en consonancia con lo propuesto en la Carta Global para la Salud Pública $(11,12)$.

( "Es importante la comunicación entre Gobierno, agencias, industria y sociedad.”, especialmente en situaciones donde más de una organización puede tener jerarquía o prelación sobre otras, estableciendo una cadena de mando compleja que puede convertirse en un asunto difícil en términos de las medidas de seguridad y de salud ocupacional de los trabajadores, o por situaciones de crítica, estigmatización y discriminación laborales debido a la asociación entre "razón social" y "responsabilidad frente a la catástrofe" $(2,4,12)$.

— "El respeto y el buen trato de datos e información son esenciales para realizar investigaciones, sin importar su propósito o alcance." lo que equivale, en primera instancia, al trazado, acuerdo de partes y seguimiento de los planes de respuesta a emergencias y desastres (Japón cuenta con su 'Plan Nacional de Respuesta' - Bosai Kihon Keikaku) y, en segunda, a la realización periódica de estudios de control y actualizaciones de los datos ya recogidos (4), lo cual es también una forma válida de investigación en salud. $\square \quad$ "Se precisa un equilibrio entre personal y maquinaria, pues el tiempo previsto para finalizar limpieza y cerrar planta es de 20 a 30 años"; balancear las ventajas y limitaciones de diferentes contramedidas o herramientas empleadas, que pueden ser incluso contraproducentes y generar demoras en un proceso que necesita ser ágil per se (por ejemplo, el uso de las máscaras de respiración), y a adaptar la noción de "emergencia" o de "catástrofe" en función de la larga vida media de varios isótopos que escaparon los diferentes niveles de seguridad de la planta y que llegaron al medio ambiente $(2,4)$.

(1) "Evitar incorporar técnicas o tecnologías industriales desconocidas o foráneas" sin valorar costo, implicaciones o posibles riesgos de salud individual o pública; en Colombia ya se están observando los debates, peligros y efectos a corto y mediano plazo de prácticas como la extracción de petróleo o gas mediante fractura hidráulica o fracking (13), o la minería no planeada o ilegal y subsecuente contaminación con metales pesados (14).

Conflicto de intereses: el autor declara que no posee conflictos de intereses

\section{Referencias}

1. Thielen H. The Fukushima Daiichi nuclear accident--an overview. Health Phys. 2012;103(2):169-74.

2. The Fukushima Daiichi Nuclear Accident. Japan AESo, (ed.). Tokyo: Springer; 2015.

3. Aliyu AS, Evangeliou N, Mousseau TA, Wu J, Ramli AT. An overview of current knowledge concerning the health and environmental consequences of the Fukushima Daiichi Nuclear Power Plant (FDNPP) accident. Environ Int. 2015;85:213-28.

4. Hiraoka K, Tateishi S, Mori K. Review of health issues of workers engaged in operations related to the accident at the Fukushima Daiichi Nuclear Power Plant. J Occup Health. 2015;57(6):497-512.

5. Hernández EJ, Camargo ZM, Martínez PMT. Impact of 5S on productivity, quality, organizational climate and industrial safety in Caucho Metal Ltda. Ingeniare Rev Chil Ing. 2005;23(1):10717.

6. Scholl C, Lieberz W, Jansing P, Kupper T. The Fukushima accident and travel medicine--analysis and recommendations. Travel Med Infect Dis. 2013;11(3):139-45.

7. Hasegawa A, Tanigawa K, Ohtsuru A, Yabe H, Maeda M, Shigemura J, et al. Health effects of radiation and other health problems in the aftermath of nuclear accidents, with an emphasis on Fukushima. Lancet. 2015;386(9992):479-88.

8. Ohtsuru A, Tanigawa K, Kumagai A, Niwa O, Takamura $\mathbf{N}$, Midorikawa S, et al. Nuclear disasters and health: lessons learned, challenges, and proposals. Lancet. 2015;386(9992):48997.

9. Eddy C, Sase E. Implications of the Fukushima Nuclear Disaster: Man-Made Hazards, Vulnerability Factors, and Risk to Environmental Health. J Environ Health. 2015;78(1):26-32.

10.Leppold C, Tanimoto T, Tsubokura M. Public health after a nuclear disaster: beyond radiation risks. Bull World Health 
Organ. 2016;94(11):859-60.

11.Lomazzi M. A Global Charter for the Public's Health-the public health system: role, functions, competencies and education requirements. Eur J Public Health. 2016;26(2):210-2.

12.Shimura T, Yamaguchi I, Terada H, Robert Svendsen E, Kunugita N. Public health activities for mitigation of radiation exposures and risk communication challenges after the Fukushima nuclear accident. J Radiat Res. 2015;56(3):422-9.

13.Paylor A. The social-economic impact of shale gas extraction: a global perspective. Third World Quarterly. 2016;38(2):340-55.

14.Casas IC, Gomez E, Rodriguez LM, Giron SL, Mateus JC. [Toward a national plan to control health effects of mercury in Colombia]. Biomedica : revista del Instituto Nacional de Salud. 2015;35 Spec:30-7. 